\title{
Comment on renal autotransplantation: A final option to preserve the kidney after an iatrogenic ureteral injury
}

\author{
Christos Damaskos ${ }^{1,2^{*}}$, Nikolaos Garmpis ${ }^{2,3^{*}}$, Konstantinos Nikolettos ${ }^{4}$, Alexandros Patsouras ${ }^{2}$, \\ Dimitrios Schizas ${ }^{5}$, Anna Garmpi ${ }^{6}$, Vasiliki E. Georgakopoulou ${ }^{7}$, Athanasios Syllaios ${ }^{5}$, \\ Dimitrios Dimitroulis ${ }^{3}$ \\ ${ }^{1}$ Renal Transplantation Unit, Laiko General Hospital, Athens, Greece; \\ ${ }^{2}$ N.S. Christeas Laboratory of Experimental Surgery and Surgical Research, Medical School, National and Kapodistrian University \\ of Athens, Athens, Greece; \\ ${ }^{3}$ Second Department of Propedeutic Surgery, Laiko General Hospital, Medical School, National and Kapodistrian University \\ of Athens, Athens, Greece; \\ ${ }^{4}$ Obstetric and Gynecologic Clinic, Medical School, Democritus University of Thrace, Alexandroupolis, Greece; \\ ${ }^{5}$ First Department of Surgery, Laiko General Hospital, Medical School, National and Kapodistrian University of Athens, Athens, \\ Greece; \\ ${ }^{6}$ First Department of Propedeutic Internal Medicine, Laiko General Hospital, Medical School, National and Kapodistrian University \\ of Athens, Athens, Greece; \\ ${ }^{7}$ Department of Pulmonology, Laiko General Hospital, Athens, Greece. \\ *Equal contribution.
}

Submitted 26 May 2021; Accepted 12 July 2021

To the Editor,

Autologous Renal Transplantation (ART) since firstly described in 1963 by Hardy, has been used in various cases (1).

There are various reasons for the transplantation such as iatrogenic ureteral damage, chronic kidney pain, unresectable renal tumors or renovascular diseases $(2,3)$. Indications concerning the suitable patients for this kind of procedure are gradually increasing. Nevertheless, each case is unique, and the treatment must be personalized.

ART is a procedure with various complications. These include infections, graft failure, urine leak and renal vein thrombosis (2). However, the kidney preservation is of great clinical significance for the patient. In response to the case of Moulavasilis et al., kidney preservation was a decision made by both the patient and the multidisciplinary medical team (4). The patient was 41 years old, still capable of getting pregnant. However, she was in an advanced but not prohibitive age for reproduction with increased possibility of complications. Why to operate since there is one more functional kidney? During the second half of pregnancy, the glomerular infiltration is increased more than $40 \%$ normally (5). Chronic kidney disease or even small renal impairment can facilitate the appearance of pregnancy complications such as preeclampsia, in which albuminuria and arterial hypertension are included (6). Furthermore, arterial preeclampsia can accelerate renal damage, by causing podocyte loss, endothelial damage, and acute kidney injury (5). As a result, the preference of the patient for renal preservation, the possible desire for pregnancy as well as her good clinical state rendered surgery the final choice.

All the aforementioned complications can also occur in pregnancy after renal transplantation (7). Risk factors for complications are immunosuppressive treatment (donor transplantation), maternal proteinuria and hypertension (8). These pregnancies are associated with higher risk of both maternal and fetal complications. Acute graft rejection, preeclampsia, cesarian sections and low birth weight of fetus are common problems. It should be noticed that the possibility of graft loss is slightly higher the first 2 years postpartum, and no difference is noticed 10 years postpartum compared to nulliparous controls (7-9).

Except for renal autotransplantation, other choices also exist in patients with ureteral avulsion. Anastomosis between pelvis of the kidney and ileum constitutes the ileal ureter. It is recommended in cases with extensive ureter injuries. However, urinary infections and pain can occur in the post-surgical period (10). Furthermore, metabolic and intestinal complications are also a frequent medical entity after this type of surgery (11). Even appendix interposition has been reported in the literature (12).

Buccal mucosa onlay constitutes another choice for management of ureteral injuries. The oral mucosa is easily accessible and wet. Grafts are taken from either the inner chick or lip. It is an innovative technique, which is used the last years. It can be performed as an open surgery, laparoscopic or robot assisted. Even though the reported success of this surgery is very high, this technique should be used in larger series for final assessment (13). However, no information about the success of this surgery exists in patients who need reconstruction of the ureter more than $11 \mathrm{~cm}$ (14). Restenosis or stricture recurrence have also occurred in some cases $(13,15)$. Nephrostomy is another alternative for cases of ureteral damage. It is a minimally invasive technique which is easily performed. It is usually conducted in patients, in order to stabilize them, and does not usually constitute a permanent solution. The morbidity is very low, less than $0.04 \%$ and com-

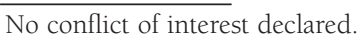


plications include colon perforation or bleeding. Antibiotic usage before the implementation of the nephrostomy should be initiated (16).

Finally, nephrectomy is another choice in patients with extensive ureteral damage. The decision of the patient and the difficulty of a possible ureter reconstruction render laparoscopic nephrectomy a possible choice (17). In addition, this procedure is safer, with less complication especially in patients with other comorbidities. On the other hand, the patient has only one functional kidney.

Various surgical techniques exist for the management of ureteral avulsion. ART is a medical intervention, which can be both beneficial and harmful. Medical expertise is required, and its use should be implemented when conventional therapeutic methods have already been considered. The reason, time and type of surgery are very important for the clinical outcome of the patient. Thus, all types of management can be considered both right and wrong, depending on the final clinical outcome. ART is a challenge.

\section{REFERENCES}

1. Hardy JD, Eraslan S. Autotransplantation of the kidney for high ureteral injury. J Urol. 1963; 90:563-574.

2. Vrakas G, Sullivan M. Current review of renal autotransplantation in the UK. Curr Urol Rep. 2020; 21:33.

3. Liu LH, Chen Z, Xiong YY, et al. Clinical application of renal autotransplantation in complex urological disease]. Zhonghua Yi Xue Za Zhi. 2019; 99:907-911.

4. Moulavasilis N, Katafigiotis I, Staios D, et al. Renal autotransplantation: A final option to preserve the kidney after an iatrogenic ureteral injury. Arch Ital Urol Androl. 2020; 91:263-264.

5. Cornelis T, Odutayo A, Keunen J, Hladunewich M. The kidney in normal pregnancy and preeclampsia. Semin Nephrol. 2011; 31:4-14.

6. Kattah A. Preeclampsia and kidney disease: Deciphering cause and effect. Curr Hypertens Rep. 2020; 22:91.

7. Deshpande NA, James NT, Kucirka LM, et al. Pregnancy outcomes in kidney transplant recipients: A systematic review and meta-analysis. Am J Transplant. 2011; 11:2388-2404.

8. van Buren MC, Schellekens A, Groenhof TKJ, et al. Long-term graft survival and graft function following pregnancy in kidney transplant recipients: A systematic review and meta-analysis. Transplantation. 2020; 104:1675-1685.

9. Sibanda N, Briggs JD, Davison JM, et al. Pregnancy after organ transplantation: A report from the UK Transplant pregnancy registry. Transplantation. 2007; 83:1301-1307.

10. Sevinc C, Balaban M, Ozkaptan O, et al. The management of total avulsion of the ureter from both ends: Our experience and literature review. Arch Ital Urol Androl. 2016; 88:97-100.

11. Kocot A, Kalogirou C, Vergho D, Riedmiller H. Long-term results of ileal ureteric replacement: A 25-year single-centre experience. BJU Int. 2017; 120:273-279.

12. Dagash H, Sen S, Chacko J, et al. The appendix as ureteral substitute: A report of 10 cases. J Pediatr Urol. 2008; 4:14-19.

13. Xiong S, Wang J, Zhu W, et al. Onlay repair technique for the management of ureteral strictures: A comprehensive review. Biomed Res Int. 2020; 2020:6178286.

14. Zhao LC, Yamaguchi Y, Bryk DJ, et al. Robot-assisted ureteral reconstruction using buccal mucosa. Urology. 2015; 86:634-638.

15. Arora S, Campbell L, Tourojman M, et al. Robotic buccal mucosal graft ureteroplasty for complex ureteral stricture. Urology. 2017; 110:257-258.

16. Zagoria RJ, Dyer RB. Do's and don't's of percutaneous nephrostomy. Acad Radiol. 1999; 6:370-377.

17. Ordon M, Schuler TD, Honey RJ. Ureteral avulsion during contemporary ureteroscopic stone management: "The scabbard avulsion". J Endourol. 2011; 25:1259-1262.

\section{Correspondence}

Christos Damaskos, MD, MSc, PhD (Corresponding Author)

x_damaskos@yahoo.gr

Renal Transplantation Unit, Laiko General Hospital \& N.S. Christeas

Laboratory of Experimental Surgery and Surgical Research, Medical School,

National and Kapodistrian University of Athens; 17 Agiou Thoma Street,

Athens, 11527 (Greece)

Nikolaos Garmpis, MD, MSc, PhD

N.S. Christeas Laboratory of Experimental Surgery and Surgical Research, Medical School, National and Kapodistrian University of Athens \& Second Department of Propedeutic Surgery, Laiko General Hospital, Medical School, National and Kapodistrian University of Athens, Athens, (Greece)

Konstantinos Nikolettos, MD

Obstetric and Gynecologic Clinic, Medical School, Democritus University of Thrace, Alexandroupolis, (Greece)

Alexandros Patsouras, MD

N.S. Christeas Laboratory of Experimental Surgery and Surgical Research, Medical School, National and Kapodistrian University of Athens, Athens (Greece)
Dimitrios Schizas, MD, PhD, Assistant Professor of Surgery

First Department of Surgery, Laiko General Hospital, Medical School, National and Kapodistrian University of Athens, Athens (Greece)

Anna Garmpi, MD

First Department of Propedeutic Internal Medicine, Laiko General Hospital, Medical School, National and Kapodistrian University of Athens, Athens

(Greece)

Vasiliki E. Georgakopoulou, MD

Department of Pulmonology, Laiko General Hospital, Athens (Greece)

Athanasios Syllaios, MD

First Department of Surgery, Laiko General Hospital, Medical School, National and Kapodistrian University of Athens, Athens (Greece)

Dimitrios Dimitroulis, MD, MSc, PhD, Professor of Surgery

Second Department of Propedeutic Surgery, Laiko General Hospital,

Medical School, National and Kapodistrian University of Athens, Athens

(Greece) 